\title{
SOME ELEMENTARY CONSEQUENCES OF PERELMAN'S CANONICAL NEIGHBORHOOD THEOREM
}

\author{
BENNETT CHOW AND PENG LU ${ }^{1}$
}

In this purely expository note, we recall a few known direct consequences of Perelman's canonical neighborhood theorem for 3-dimensional Ricci flow and compactness theorem for 3 -dimensional $\kappa$-solutions. These corollaries regard elementary properties of 3 -dimensional singularity models and $\kappa$-solutions. Throughout this note, convergence is in the pointed $C^{\infty}$ Cheeger-Gromov sense, $\cong$ denotes diffeomorphic, Rm denotes the Riemann curvature tensor, and $i \in \mathbb{N}$.

Given $\kappa>0$, a complete solution $\left(\mathcal{M}^{n}, \tilde{g}(t)\right), t \in(-\infty, 0]$, of the Ricci flow is called a $\kappa$-solution if $\tilde{g}(t), t \in(-\infty, 0]$, is nonflat with uniformly bounded nonnegative curvature operator $\mathrm{Rm} \geq 0$ and $\kappa$-noncollapsed at all scales.

Corollary 1. If $\left(\mathcal{M}^{3}, g(t)\right), t \in[0, T), T<\infty$, is a singular solution to the Ricci flow on a closed 3-manifold, then for any $\left(x_{i}, t_{i}\right) \in \mathcal{M} \times[0, T)$ with scalar curvature $R_{i} \doteqdot R\left(x_{i}, t_{i}\right) \rightarrow \infty$, there exists a subsequence of $\left(\mathcal{M}, \tilde{g}_{i}(t),\left(x_{i}, 0\right)\right)$, where $\tilde{g}_{i}(t) \doteqdot R_{i} g\left(t_{i}+R_{i}^{-1} t\right)$, converging to a $\kappa$-solution $\left(\mathcal{M}_{\infty}^{3}, g_{\infty}(t),\left(x_{\infty}, 0\right)\right)$. In particular, $\left|\mathrm{Rm}_{g_{\infty}(t)}\right| \leq C$ on $\mathcal{M}_{\infty} \times(-\infty, 0]$ for some $C<\infty$.

Proof. By Perelman's improved no local collapsing theorem [3] (see also [4]), there exists $\kappa>0$ such that if $x_{0} \in \mathcal{M}, t_{0} \in[0, T)$, and $r_{0} \in(0,1)$ are such that $R \leq r_{0}^{-2}$ in $B_{g\left(t_{0}\right)}\left(x_{0}, r_{0}\right)$, then $\frac{\operatorname{Vol}_{g\left(t_{0}\right)} B_{g\left(t_{0}\right)}\left(x_{0}, r\right)}{r^{n}} \geq \kappa$ for $r \in\left(0, r_{0}\right]$. 2 Perelman's canonical neighborhood Theorem 12.1 in [3] (see also [1]) says that for $j \in \mathbb{N}$, there exists $r_{j} \in(0,1]$ such that if $i_{j}$ is chosen large enough so that $R_{i_{j}} \geq r_{j}^{-2}$, then $\tilde{g}_{i_{j}}(t)$ on $B_{\tilde{g}_{i_{j}}(0)}\left(x_{i_{j}}, j^{1 / 2}\right) \times[-j, 0]$ is $\frac{1}{j}$-close to the corresponding subset of a $\kappa$-solution $\left(\mathcal{N}_{j}^{3}, h_{j}(t)\right)$ centered at $y_{j} \in \mathcal{N}_{j}$. From Perelman's compactness Theorem 11.7 in [3] for 3-dimensional $\kappa$-solutions and since $\lim _{j \rightarrow \infty} R_{h_{j}}\left(y_{j}, 0\right)=$ $\lim _{j \rightarrow \infty} R_{\tilde{g}_{i_{j}}}\left(x_{i_{j}}, 0\right)=1$, we have that $\left(\mathcal{N}_{j}, h_{j}(t),\left(y_{j}, 0\right)\right)$ subconverges to a $\kappa$ solution $\left(\mathcal{N}_{\infty}^{3}, h_{\infty}(t),\left(y_{\infty}, 0\right)\right), t \in(-\infty, 0]$. Since $\frac{1}{j} \rightarrow 0$ as $j \rightarrow \infty$, we conclude that $\left(\mathcal{M}, \tilde{g}_{i_{j}}(t),\left(x_{i_{j}}, 0\right)\right)$ subconverges to $\left(\mathcal{N}_{\infty}, h_{\infty}(t),\left(y_{\infty}, 0\right)\right), t \in(-\infty, 0]$.

A singularity model of a singular solution $\left(\mathcal{M}^{n}, g(t)\right), t \in[0, T), T<\infty$, on a closed manifold is a complete nonflat ancient solution which is the limit of $g_{i}(t) \doteqdot$ $K_{i} g\left(t_{i}+K_{i}^{-1} t\right)$ for some $t_{i} \rightarrow T$ and $K_{i} \rightarrow \infty$.

Corollary 2. Any 3-dimensional singularity model must be a $\kappa$-solution.

Proof. Let $\left(\mathcal{M}_{\infty}^{3}, g_{\infty}(t)\right), t \in(-\infty, 0]$, be a singularity model of a singular solution $\left(\mathcal{M}^{3}, g(t)\right), t \in[0, T), T<\infty$, on a closed 3 -manifold. Then there exist $\left(x_{i}, t_{i}\right)$ and $K_{i} \rightarrow \infty$ such that $\left(\mathcal{M}, g_{i}(t),\left(x_{i}, 0\right)\right)$ converges to $\left(\mathcal{M}_{\infty}^{3}, g_{\infty}(t),\left(x_{\infty}, 0\right)\right)$, where $g_{\infty}(t)$ is nonflat. Let $R_{i} \doteqdot R_{g}\left(x_{i}, t_{i}\right)$. Since $\lim _{i \rightarrow \infty} K_{i}^{-1} R_{i}=R_{g_{\infty}}\left(x_{\infty}, 0\right) \doteqdot c$

\footnotetext{
${ }^{1}$ Addresses. Bennett Chow: Math. Dept., UC San Diego; Peng Lu: Math. Dept., U of Oregon.
}

${ }^{2}$ Thus noncompact singularity models with $R$ bounded have at least linear volume growth. 
exists, where $c>0$ by the strong maximum principle, we also have that $\tilde{g}_{i}(t) \doteqdot$ $R_{i} g\left(t_{i}+R_{i}^{-1} t\right)$ converges to $\left(\mathcal{M}_{\infty}, \tilde{g}_{\infty}(t),\left(x_{\infty}, 0\right)\right), t \in(-\infty, 0]$, where $\tilde{g}_{\infty}(t)=$ $c g_{\infty}\left(c^{-1} t\right)$. By Corollary 1, there exists $\left\{i_{j}\right\}$ such that $\left(\mathcal{M}, \tilde{g}_{i_{j}}(t),\left(x_{i_{j}}, 0\right)\right)$ converges to a $\kappa$-solution $\left(\mathcal{N}_{\infty}^{3}, h_{\infty}(t),\left(y_{\infty}, 0\right)\right), t \in(-\infty, 0]$. Hence $\left(\mathcal{M}_{\infty}, \tilde{g}_{\infty}(t)\right)$ is isometric to $\left(\mathcal{N}_{\infty}, h_{\infty}(t)\right)$ on $(-\infty, 0]$. Thus $g_{\infty}(t)=c^{-1} \tilde{g}_{\infty}(c t)$ is a $\kappa$-solution.

Let $\left(\mathcal{N}^{3}, h\right)$ be a complete Riemannian 3-manifold. Given $\varepsilon>0$ and $p \in \mathcal{N}$ with $R(p)>0$, a geodesic ball $B\left(p, \varepsilon^{-1} r\right)$ is called an $\varepsilon$-neck if $r^{-2} h$ on $B\left(p, \varepsilon^{-1} r\right)$ is $\varepsilon$-close in the $C^{\left\lceil\varepsilon^{-1}\right\rceil+1}$-topology (here $\left\lceil\varepsilon^{-1}\right\rceil$ denotes the least integer $\geq \varepsilon^{-1}$ ) to a piece of the unit cylinder $g_{\mathrm{cyl}}=g_{\mathcal{S}^{2}}+d u^{2}$ on $\mathcal{S}^{2} \times \mathbb{R}$.

We have the following (which may also be proved using Corollary 9.88 in [2]).

Corollary 3. The asymptotic cone of a noncompact orientable 3 -dimensional $\kappa$ solution must be either a line or a half-line.

Proof. Let $\left(\mathcal{M}^{3}, g(t)\right), t \in(-\infty, 0]$, be a noncompact orientable 3-dimensional $\kappa$-solution. Since $\mathrm{Rm} \geq 0$, by the strong maximum principle and Hamilton's classification of 2-dimensional $\kappa$-solutions, $\left(\mathcal{M}^{3}, g(t)\right)$ is isometric to:

(i) $\mathcal{S}^{2} \times \mathbb{R}$ or $\left(\mathcal{S}^{2} \times \mathbb{R}\right) / \mathbb{Z}_{2}$, where $\mathcal{S}^{2}$ is the shrinking round 2-sphere, or

(ii) a noncompact $\kappa$-solution with $\mathrm{Rm}>0$ and $\mathcal{M} \cong \mathbb{R}^{3}$.

In case (i), the asymptotic cone of $(\mathcal{M}, g(t))$ is either a line or a half-line. In case (ii) it suffices to prove the asymptotic cone of $g(0)$, which exists since sect $\geq 0$, is a half-line; here sect denotes the sectional curvature.

Claim 1. For any $\varepsilon>0$ there exists an $\varepsilon$-neck $\mathcal{N}_{\varepsilon}$ contained in $\left(\mathcal{M}^{3}, g(0)\right)$.

Recall from Proposition 11.4 of [3] that the asymptotic scalar curvature ratio $\operatorname{ASCR}(g(t))=\infty$ for $t \in(-\infty, 0]$. Hence, by dimension reduction for noncompact $\kappa$-solutions with ASCR $=\infty$, there exists a sequence $\left\{x_{i}\right\}$ in $\mathcal{M}$ such that $g_{i}(t)=$ $R_{i} g\left(R_{i}^{-1} t\right)$, where $R_{i} \doteqdot R_{g}\left(x_{i}, 0\right)$, on $\mathcal{M} \times(-\infty, 0]$ and based at $\left(x_{i}, 0\right)$, converges to the product of $\mathbb{R}$ with a 2 -dimensional $\kappa$-solution, which must be the shrinking round $\mathcal{S}^{2}$. The existence of $\varepsilon$-necks in $(\mathcal{M}, g(t))$, for any $\varepsilon>0$ and any $t \in(-\infty, 0]$, now follows from the definition of convergence. This proves Claim 1.

Since $\mathcal{N}_{\varepsilon} \cong \mathcal{S}^{2} \times \mathbb{R}$ and $\partial \mathcal{N}_{\varepsilon}$ is embedded in $\mathcal{M} \cong \mathbb{R}^{3}$, by the smooth Schönflies theorem we have that $\mathcal{M}-\mathcal{N}_{\varepsilon}$ has exactly two components, a compact component $\mathcal{B}_{\varepsilon}$ diffeomorphic to a closed 3-ball and a noncompact component $\mathcal{C}_{\varepsilon} \cong \mathcal{S}^{2} \times[0,1)$.

Since $\mathcal{N}_{\varepsilon}$ is an $\varepsilon$-neck, there exist an embedding $\psi_{\varepsilon}: \mathcal{S}^{2} \times\left[-\varepsilon^{-1}+4, \varepsilon^{-1}-4\right] \rightarrow$ $\mathcal{N}_{\varepsilon}$ and $r_{\varepsilon}>0$ such that $r_{\varepsilon}^{-2} \psi_{\varepsilon}^{*} g(0)$ is $\varepsilon$-close in the $C^{\left[\varepsilon^{-1}\right\rceil+1}$-topology to $g_{\text {cyl }}$. We may assume that $\psi_{\varepsilon}\left(\mathcal{S}^{2} \times\left\{-\frac{1}{\varepsilon}+4\right\}\right)$ is closer to $\partial \mathcal{B}_{\varepsilon}$ and $\psi_{\varepsilon}\left(\mathcal{S}^{2} \times\left\{\frac{1}{\varepsilon}-4\right\}\right)$ is closer to $\partial \mathcal{C}_{\varepsilon}$. Since $g(0)$ has bounded curvature, $r_{\varepsilon} \geq c>0$, independent of $\varepsilon$ small.

Claim 2. From now on, fix $O \in \mathcal{M}$. For $\varepsilon>0$ sufficiently small, $O \in \mathcal{B}_{\varepsilon}$.

Since $\lim _{\varepsilon \rightarrow 0} \max _{x \in \mathcal{N}_{\varepsilon}}\left(\min \left\{\operatorname{sect}_{g(0)}\left(P_{x}\right): P_{x}\right.\right.$ is a 2-plane at $\left.\left.x\right\}\right)=0\left(g_{\text {cyl }}\right.$ has a 0 sectional curvature everywhere) and since $\operatorname{Rm}_{g(0)}(O)>0$, for $\varepsilon>0$ small we have that $O \notin \mathcal{N}_{\varepsilon}$. If the claim is false, then there exist $\varepsilon_{i} \searrow 0$ such that $O \in \mathcal{C}_{\varepsilon_{i}}$ for all $i$. We may pass to a subsequence $\left\{k_{i}\right\}$ with (the $\mathcal{N}_{\varepsilon_{k_{i}}}$ are pairwise disjoint)

$$
\mathcal{N}_{\varepsilon_{k_{i}}} \subset \mathcal{C}_{\varepsilon_{k_{j}}} \text { for } j<i \text {. }
$$

Indeed, suppose we have chosen $1 \doteqdot k_{1}<\cdots<k_{i-1}$. Since $\mathcal{K}_{i} \doteqdot \bigcup_{j<i}\left(\overline{\mathcal{M}-\mathcal{C}_{\varepsilon_{k_{j}}}}\right)$ is compact, $\operatorname{sect}_{g(0)}$ has a positive lower bound on $\mathcal{K}_{i}$. By this and $\lim _{i \rightarrow \infty} \varepsilon_{i}=0$, we conclude that there exists $k_{i}>k_{i-1}$ such that $\mathcal{N}_{\varepsilon_{k_{i}}} \cap \mathcal{K}_{i}=\varnothing$, which implies (11). 
By again using the smooth Schönflies theorem, for each $i$ and $j$ with $j<i$ we have $\mathcal{C}_{\varepsilon_{k_{j}}}-\mathcal{N}_{\varepsilon_{k_{i}}} \doteqdot \mathcal{K}_{i, j} \cup \mathcal{L}_{i, j}$, where $\mathcal{K}_{i, j} \cong \mathcal{S}^{2} \times[0,1)$ has compact closure in $\mathcal{M}$ and $\mathcal{L}_{i, j} \cong \mathcal{S}^{2} \times[0,1)$ satisfies $\overline{\mathcal{L}_{i, j}}=\mathcal{L}_{i, j}$. Since $\mathcal{M}^{3} \cong \mathbb{R}^{3}$, we conclude $\mathcal{B}_{\varepsilon_{k_{j}}} \subset \mathcal{B}_{\varepsilon_{k_{i}}}$ and $\mathcal{C}_{\varepsilon_{k_{j}}} \supset \mathcal{C}_{\varepsilon_{k_{i}}}$ for all $j<i$. Claim 2 follows from:

Subclaim. $\bigcap_{i \in \mathbb{N}} \mathcal{C}_{\varepsilon_{k_{i}}}=\varnothing$.

Fix $p \in \mathcal{B}_{\varepsilon_{k_{1}}}$, so that $p \in \mathcal{B}_{\varepsilon_{k_{i}}}$ for all $i$. Suppose the subclaim is false; then there exists $x \in \mathcal{C}_{\varepsilon_{k_{i}}}$ for all $i$. Let $\gamma$ be a minimal geodesic from $p$ to $x$ with respect to $g(0)$. Then $\gamma$ must pass from one end of $\mathcal{N}_{\varepsilon_{k_{i}}}$ to the other end. Hence, for $i$ large,

$$
d_{g(0)}(p, x)=\mathrm{L}_{g(0)}(\gamma) \geq \frac{1}{2} \operatorname{diam}_{g(0)}\left(\mathcal{N}_{\varepsilon_{k_{i}}}\right) \geq \frac{1}{2} \varepsilon_{k_{i}}^{-1} r_{\varepsilon_{k_{i}}} \geq \frac{c}{2} \varepsilon_{k_{i}}^{-1},
$$

where $c>0$ is independent of $i$. The subclaim follows from $\varepsilon_{k_{i}}^{-1} \rightarrow \infty$.

Now let $\operatorname{Ray}_{\mathcal{M}}(O)$ denote the space of unit speed rays emanating from $O$ in $\left(\mathcal{M}^{3}, g(0)\right)$. We have the pseudo-metric $\tilde{d}_{\infty}\left(\gamma_{1}, \gamma_{2}\right) \doteqdot \lim _{s, t \rightarrow \infty} \tilde{\measuredangle} \gamma_{1}(s) O \gamma_{2}(t) \in$ $[0, \pi]$ on $\operatorname{Ray}_{\mathcal{M}}(O)$, for $\gamma_{1}, \gamma_{2} \in \operatorname{Ray}_{\mathcal{M}}(O)$ and where $\tilde{\measuredangle}$ is the Euclidean comparison angle. The asymptotic cone of $(\mathcal{M}, g(0))$ is isometric to the Euclidean metric cone Cone $\left(\mathcal{M}(\infty), d_{\infty}\right)$, where $\left(\mathcal{M}(\infty), d_{\infty}\right)$ is the quotient metric space induced by $\left(\operatorname{Ray}_{\mathcal{M}}(O), \tilde{d}_{\infty}\right)$. Thus, the conclusion that the asymptotic cone of $(\mathcal{M}, g(0))$ is a half-line shall follow from showing that for all $\gamma_{1}, \gamma_{2} \in \operatorname{Ray}_{\mathcal{M}}(O), \tilde{d}_{\infty}\left(\gamma_{1}, \gamma_{2}\right)=0$.

Since $O \in \mathcal{B}_{\varepsilon}$ for $\varepsilon>0$ sufficiently small, we have for such $\varepsilon$ that any $\gamma \in$ $\operatorname{Ray}_{\mathcal{M}}(O)$ passes from one end of $\mathcal{N}_{\varepsilon}$ to the other end.

Claim 3. For any $\varepsilon>0$ sufficiently small, we have that any $\gamma \in \operatorname{Ray}_{\mathcal{M}}(O)$ intersects $\psi_{\varepsilon}\left(\mathcal{S}^{2} \times\left\{\varepsilon^{-1}-4\right\}\right)$ at exactly one point, which we define to be $\gamma\left(t_{\gamma, \varepsilon}\right)$.

This follows from the facts that rays minimize and that the geometry of any $\varepsilon$-neck is, after rescaling, $\varepsilon$-close to that of the unit cylinder of length $2 \varepsilon^{-1}$.

We have $\lim _{t \rightarrow \infty} \frac{d_{g(0)}\left(\gamma_{1}(a t), \gamma_{2}(b t)\right)}{t}=\left(a^{2}+b^{2}-2 a b \cos \left(\tilde{d}_{\infty}\left(\gamma_{1}, \gamma_{2}\right)\right)\right)^{1 / 2}$. Hence $\tilde{d}_{\infty}\left(\gamma_{1}, \gamma_{2}\right)=0$ if and only if $\lim _{t \rightarrow \infty} \frac{d_{g(0)}\left(\gamma_{1}(t), \gamma_{2}(t)\right)}{t}=0$. Now $\tilde{d}_{\infty}\left(\gamma_{1}, \gamma_{2}\right)=0$ follows from:

Claim 4. For any $\gamma_{1}, \gamma_{2} \in \operatorname{Ray}_{\mathcal{M}}(O), \lim _{\varepsilon \rightarrow 0} \frac{d_{g(0)}\left(\gamma_{1}\left(t_{\gamma_{1}, \varepsilon}\right), \gamma_{2}\left(t_{\gamma_{2}, \varepsilon}\right)\right)}{\min \left\{t_{\gamma_{1}, \varepsilon}, t_{\gamma_{2}, \varepsilon}\right\}}=0$.

Since $\mathcal{N}_{\varepsilon}$ is an $\varepsilon$-neck and the diameter of a round 2-sphere of radius $r$ is $\pi r$, we have that $d_{g(0)}\left(\gamma_{1}\left(t_{\gamma_{1}, \varepsilon}\right), \gamma_{2}\left(t_{\gamma_{2}, \varepsilon}\right)\right) \leq 2 \pi r_{\varepsilon}$ for $\varepsilon$ sufficiently small. Since $\gamma_{1}$ and $\gamma_{2}$ are rays emanating from the same point, this implies $\left|t_{\gamma_{1}, \varepsilon}-t_{\gamma_{2}, \varepsilon}\right| \leq 2 \pi r_{\varepsilon}$. Since $\left.\gamma_{1}\right|_{\left[0, t_{\left.\gamma_{1}, \varepsilon\right]}\right]}$ and $\left.\gamma_{2}\right|_{\left[0, t_{\left.\gamma_{2}, \varepsilon\right]}\right.}$ both intersect $\psi_{\varepsilon}\left(\mathcal{S}^{2} \times\left\{-\varepsilon^{-1}\right\}\right)$ and $\psi_{\varepsilon}\left(\mathcal{S}^{2} \times\left\{\varepsilon^{-1}-4\right\}\right)$, we have $\min \left\{t_{\gamma_{1}, \varepsilon}, t_{\gamma_{2}, \varepsilon}\right\} \geq \varepsilon^{-1} r_{\varepsilon}$. Claim 4 follows easily.

\section{REFERENCES}

[1] Kleiner, Bruce; Lott, John. Notes on Perelman's papers. Geom. Topol. 12 (2008), 2587-2855.

[2] Morgan, John; Tian, Gang. Ricci flow and the Poincaré conjecture. Clay Mathematics Monographs, 3, AMS, Providence, RI, 2007.

[3] Perelman, Grisha. The entropy formula for the Ricci flow and its geometric applications. arXiv:math.DG/0211159

[4] Topping, Peter. Diameter control under Ricci flow. Comm. Anal. Geom. 13 (2005), 1039-1055. 